\title{
Image-based measurements of degree of saturation
}

\author{
Ferran Parera ${ }^{1,2^{*}}$, Núria Pinyol ${ }^{1,2}$, Eduardo Alonso ${ }^{1,2}$ \\ ${ }^{1}$ Centre de Metodes Numerics en Enginyeria (CIMNE), Barcelona, Spain \\ ${ }^{2}$ Department of Civil and Environmental Engineering. Universitat Politècnica de Catalunya (UPC), Barcelona, Spain
}

\begin{abstract}
A method based on SWIR images is proposed for measuring the degree of saturation in soils. This method is especially suitable for use in experiments conducted in the facilities of a common geotechnical laboratory. The method uses images on the short-wave infrared (SWIR) (wavelength: 1400$1550 \mathrm{~nm}$ ), which is highly absorbed by water compared with the other bands of the light spectrum (visible and near-infrared). The paper presents the basis of the method and the procedure to measure the degree of saturation in a massive and non-invasive way. A relation curve to transform the pixel intensity values to the degree of saturation is provided for samples of a beach sand. The non-linearity of this curve is discussed and related to the optical path length.
\end{abstract}

\section{Introduction}

The measurements of the degree of saturation and other related variables (water content and suction) are important in the laboratory experiments with unsaturated soils to understand their behaviour. The size and type of experiments are determined by the dimensions and devices of the geotechnical laboratory in which they are performed.

Traditionally, the degree of saturation is measured by sensors that collect discrete data in localized volumes. These devices measurements are highly reliable due to the long experience in their use and the improvements that have been carried out. However, they present some limitations due their isolated measurements. The distribution of the degree of saturation in the rest of areas of the experiments has to be inferred or interpolated, which make more difficult to obtain the idea of the general behaviour of the soil. Moreover, the place where there is interest in knowing the degree of saturation is not always easy to predefine before the experiment.

To overcome the discrete data limitations, there are alternative methods. These methods are based on the interaction of a flux of particles (photons, electrons, or neutrons) with the unsaturated soils. The reflected or traversed particles constitute a $2 \mathrm{D}$ or $3 \mathrm{D}$ image of the degree of saturation distribution.

The neutron radiography and tomography are based on the attenuation of a flux of neutrons by the hydrogen that constitutes the water molecule $[1,2]$. This technique provides a detailed 3D distribution that enables calculate precisely the hydraulic properties of granular media. The main limitation of this method is the induced radioactivity hazard, which limits the use of this technique to specialized laboratories with isolated chambers. The size of these chambers also limits the sample size to few centimetres.

The geophysical methods are another alternative to obtain the distribution of the degree of saturation. The most relevant methods are the electrical resistivity tomography and the ground-penetration radar. The first one uses the electric current and the difference in the resistivity to its pass between the soil grains and the water [3, 4]. The ground-penetrating radar uses the microwaves and the difference between the velocity of propagation in soil and water of these electromagnetic waves $[5,6]$.

The main objective is to obtain a flexible method to measure the degree of saturation distribution in any common geotechnical laboratory. This requirement imposes that the method must be safe for biological tissue, that excludes the neutron radiography, and other methods that use the ultraviolet area of the electromagnetic spectrum. On the other side, the geophysical methods that use the area of the electromagnetic spectrum with long wavelengths, like ground-penetrating radar, are not suitable for the size of the samples tested in laboratory. The length of these waves physically limits the resolution that can be reached (Figure 1).

Taking that conditions into account, the suitable area of the electromagnetic spectrum to be used in the geotechnical laboratory measurements should be in the visual, near-infrared (NIR), or short-wave infrared (SWIR) areas. This study analyses the physical concepts behind the electromagnetic and unsaturated soil interactions. It correlates these concepts with the spectral tomography results from other authors to define the optimal range for the degree of saturation measurement. The soil sample images obtained on this band of the electromagnetic spectrum are used to obtain an

Corresponding author: ferran.parera@upc.edu 
experimental relation curve between the degree of saturation and the pixel intensity. This curve can be used to obtain the degree of saturation distribution from images.

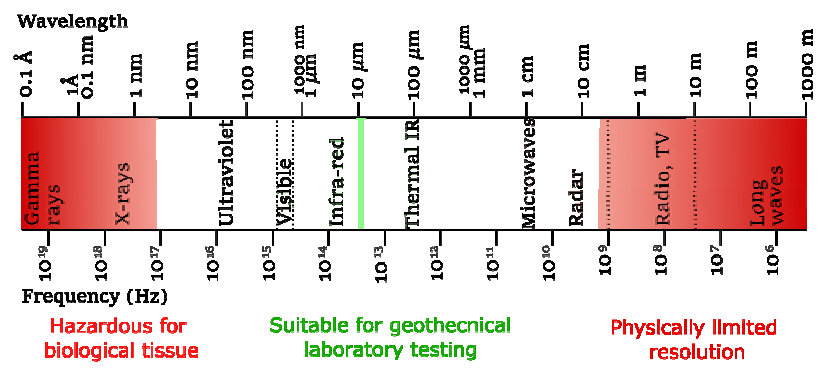

Fig. 1. Full electromagnetic spectrum. The most suitable wavelengths (frequencies) for geotechnical laboratory measurements are marked in green.

\section{Physical principles}

The electromagnetic radiation is a concept that includes the full spectrum of the common term light, which goes from the Gamma rays to further than the Radio waves. The electromagnetic waves (EM waves) that constitute the radiation are defined by their frequency or wavelength, both concepts are intrinsically bound and define the EM wave energy.

The EM waves across the spectrum interact with the matter depending on its frequency and the composition of the object. The nature of the different types of interaction lies in the atomic and molecular levels explained by the quantum theory [7]. This theory explains that the absorption of an EM wave by matter is determined by the stable quantum changes in the atomic and molecular energies. The absorption of an EM wave by a molecule requires that the EM wave provides the exact amount of energy to produce the transition from one quantum state to another valid quantum state.

Focussing on the areas of the EM spectrum of interest (visual, NIR, and SWIR), the interaction between EM waves and water is especially determined by the vibrational states of the water molecule. The water molecule has three vibration modes, which correspond to the quantum numbers v1, v2, v3. The mode v1 corresponds to symmetric stretch vibrations. The mode v2 corresponds to bending deformation vibrations. The mode v3 correspond to asymmetric stretch vibrations. Stable vibration excited states correspond to specific combinations of these three modes of vibration. Wavelengths that its energy coincides with one of these stable excited vibration states tend to be absorbed and retained by the water molecule with which they interact.

Figure 2 exposes the coefficient of light absorption by water depending on the wavelength of the EM wave [8]. The two most absorbent bands of the area of the EM spectrum analysed are 1400-1550 $\mathrm{nm}$ and 1800-2050 $\mathrm{nm}$. Both bands are in the SWIR area.

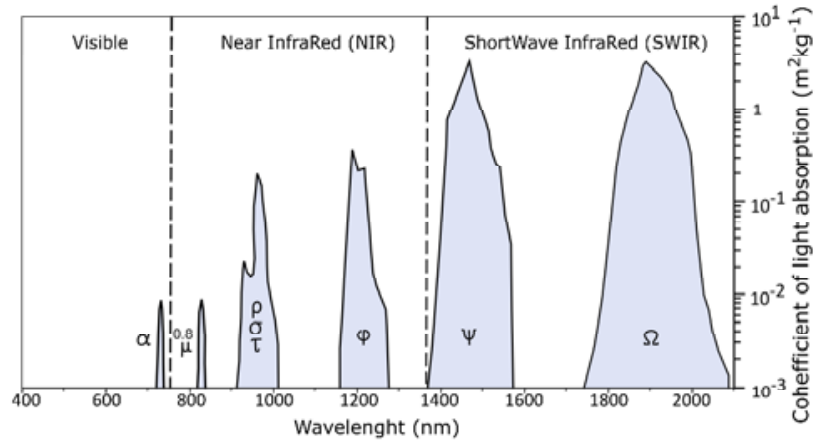

Fig. 2. Spectrum of the coefficient of light absorption by water on visible, NIR, and SWIR. Based on the figure form the book Light absorption in seawater [9].

The spectral tomography is the laboratory analysis of how the reflectance of an object changes depending on the EM wavelength of the incident light. On figure 3, Nolet et al. performed a spectral tomography on beach sand samples at different volume water content, from dry to fully saturated [9]. The graph shows that the bands of the analysed spectrum where the reflectance of the beach sand is affected by the degree of saturations are 1400$1550 \mathrm{~nm}$ and 1850-2050 nm. A correlation can be observed between the most absorbed wavelengths by water and the most sensible wavelengths to the degree of saturation of the studied soil.

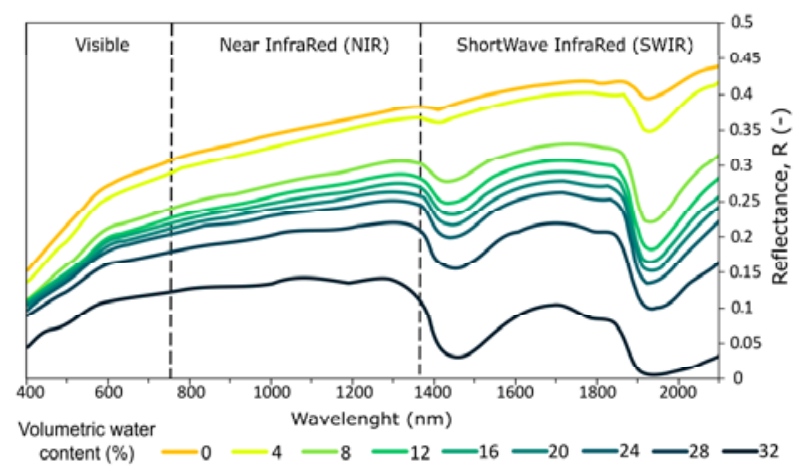

Fig. 3. Spectral tomography in a beach sand analysed the wavelength range between 400 and $2100 \mathrm{~nm}$ [9].

Several authors have performed spectral tomographies on different types of soils Lobell and [913]. The results of all these analyses has been summarized in figure 4 . The graph plots the difference between the dry and saturated soil reflectance, as a visualization of the sensitivity of each wavelength to the water content. It can be observed that there are two maximums on the sensitivity that are coincident in all the analysed soils. These correspond to the mentioned spectral bands (1400-1550 nm and 1900-2000 nm) where the water is more absolvent. The conclusion is that water controls the best bands of the analysed EM spectrum to evaluate the degree of saturation, the choice of the best bands is independent of the mineralogy or composition of the soil that is tested. 


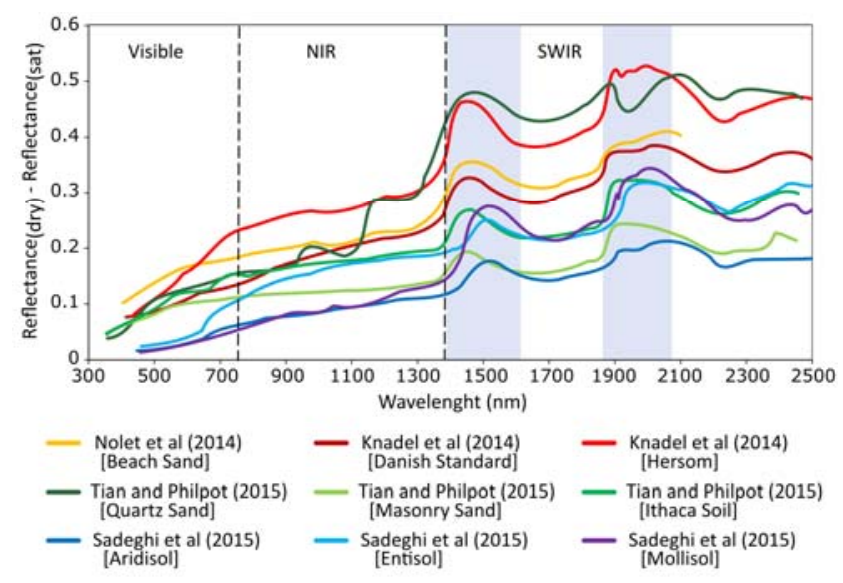

Fig. 4. Comparison the spectral tomography results of different authors. The difference between the reflectance in dry and saturated conditions is analysed for each wavelength in the range between 300 and $2500 \mathrm{~nm}$. The most sensitive ranges are shaded.

\section{Methodology}

This paper explains the method for the non-invasive measurement of the degree of saturation in granular media based on SWIR images described in Parera et al. (2019)[14]. The light reflected by the analysed surface of the unsaturated soil is captured by the camera sensor. The pixel intensity is the value that is stored on each image pixel and express the intensity of light captured by each part of the sensor at this moment.

The pixel intensity is determined by the degree of saturation, but also by the amount of incident light and the colour of the soil grains. Therefore, the effects of these factors need to be removed before translating the pixel intensity into degree of saturation A Gaussian filter is applied to the image to eliminate the effect of the different colours of the soil grains, and to eliminate sensor noises if needed. This filter implies that pixel stores the pixel intensity $(\xi)$ of the surrounding pixels pondered by their distance $\left[\left(x-x_{o}\right)^{2}+\left(y-y_{o}\right)^{2}\right]$ following the normal Gaussian distribution (Eq. 1). The size of the filter is defined by the standard deviation $(\sigma)$.

$$
\xi_{x_{o} y_{o}}=\sum \xi_{x y} \frac{1}{2 \pi \sigma^{2}} e^{-\frac{\left(x-x_{o}\right)^{2}+\left(y-y_{o}\right)^{2}}{2 \sigma^{2}}}
$$

In order to eliminate the effect of the incident light variation, the pixel intensity is normalized $\left(\xi_{\text {norm }}\right)$. The normalization is performed using the pixel intensity values for the dry $\left(\xi_{\text {dry }}\right)$ and saturated $\left(\xi_{\text {sat }}\right)$ cases (2). That normalization could also be done using distributed targets with known reflectance values.

$$
\xi_{\text {norm }}=\frac{\xi-\xi_{\text {dry }}}{\xi_{\text {sat }}-\xi_{\text {dry }}}
$$

\subsection{Degree of saturation - Pixel intensity}

The relation between the degree of saturation and the pixel intensity is analysed to provide a curve that correlates both parameters. The calibration procedure is performed using soil samples with the same dry density $\left(1390 \mathrm{~kg} / \mathrm{m}^{3}\right)$ at different degree of saturation $(0,0.02$, $0.07,0.15,0.30,0.44,0.60,0.73,1)$. A zenithal image is taken for each sample, maintaining constant the incident light and the camera distance (Fig.5).

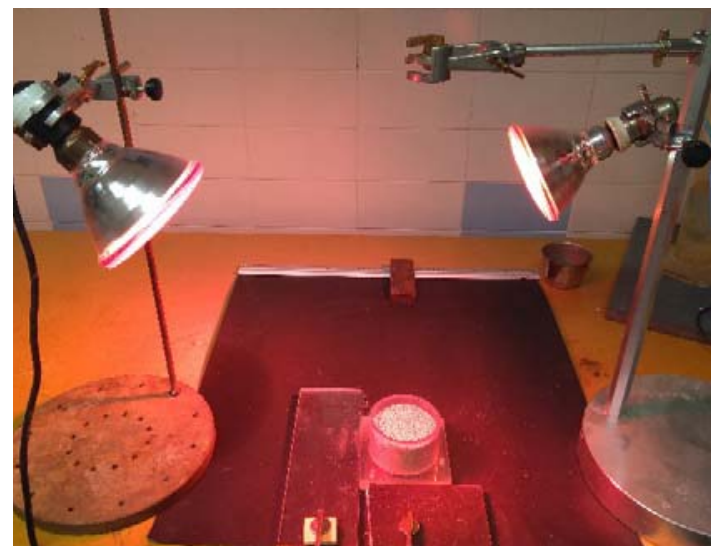

Fig. 5. Calibration procedure setup.

The Gaussian filter (Eq. 1) is applied to the images, so the average pixel intensity of each sample is obtained (Table 1). The values are plotted into a graph that correlates degree of saturation and pixel intensity for that light condition. Then the obtained curve is normalized (2) to get the relation curve independent of the incident light (Fig. 6).

Table 1. Examples of the images of the analysed soil at different degree of saturation.

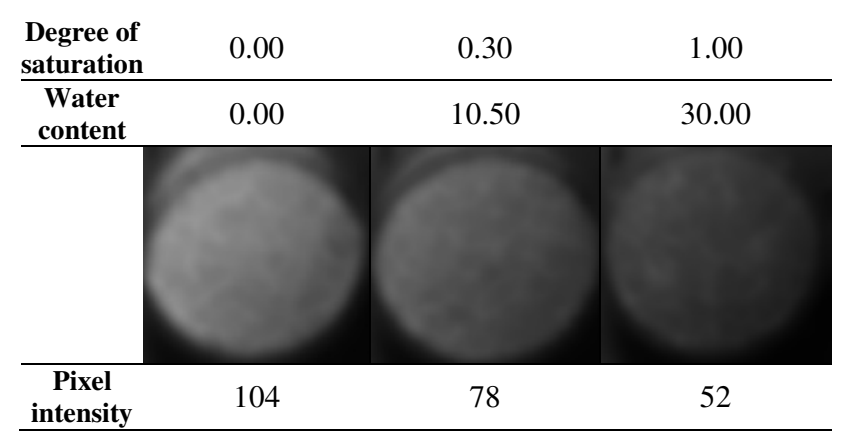

Figure 6 exposes that the relation between the pixel intensity and the degree of saturation is not linear. The reason behind the not linearity is the length of the path of light through the water, called optical path length. On dry soils, the reflectance is controlled by the soil composition, mineralogy, and granulometry. In low degree of saturation, the water sticks to each grain, forming independent fine films around each one. In that situation, the reduction in the reflectance is just due the scattering. Increasing the degree of saturation, the water attached to the grains begins to connect each other forming capillarity bridges. The increment of this water meniscus increases the optical path length, and therefore, the light has more length to be absorbed by water molecules. When the degree of saturation reaches values close to the full saturation, free water appears on the surface, even some air remains trapped between the 
grains. In that situation the reflectance of the soil is defined by the water optical properties $[9,10,15]$.

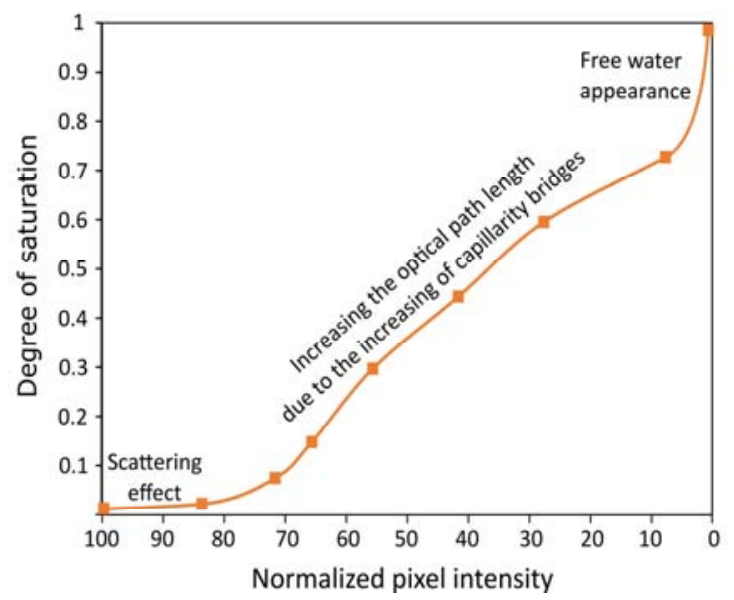

Fig. 4. Normalized pixel intensity - Degree of saturation relation curve.

\section{Conclusions}

The method described is based on the physical principles of light absorption by unsaturated soils. The proposal of the best bands of the electromagnetic spectrum to measure the degree of saturation in geotechnical laboratories is supported by the spectral tomography studies of various authors. The two optimal bands are between the wavelengths 1400-1550 nm and 1900-2000 $\mathrm{nm}$, both in the SWIR spectrum. The choice of these bands is determined by the quantum properties of water molecules, and therefore are independent of the mineralogical composition of the soil.

The degree of saturation gradually decreases the light reflected by the surface of a soil. This light is captured by the camera sensor to obtain an image of those bands reflected light. The equations necessary to eliminate other variables that influence the amount of light reflected by a soil (amount of incident light, grain mineralogy) are shown.

The relationship Degree of saturation - Pixel intensity has been found, from the images of soil samples at different degrees of saturation. The relation is not linear because the effect of the coefficient of light absorption by water depends on the optical path of light, which depends on the creation of capillarity bridges between soil grains.

\section{References}

1. L.G. Tumlinson, H. Liu, W.K. Silk, and J.W. Hopmans, Soil Science Society of America Journal, 72 (2008).

2. M. Kang, E. Perfect, C.L. Cheng, H.Z. Bilheux, J. Lee, J. Horita, and J.M. Warren, Advances in water resources, 65 (2014).

3. W. Daily, A. Ramirez, D. LaBrecque, and J. Nitao, Water Resources Research, 28 (1992).
4. Q.Y. Zhou, J. Shimada, and A. Sato, Water Resources Research, 37 (2001).

5. R.J. Greaves, D.P. Lesmes, J.M. Lee, and M.N. Toksöz, Geophysics, 61 (1996).

6. J.A. Huisman, S.S. Hubbard, J.D. Redman, and A.P. Annan, Vadose zone journal, 2 (2003).

7. M. Planck, The theory of heat radiation (1914).

8. B. Wozniak and J. Dera, Light absorption in sea water (2006).

9. C. Nolet, A. Poortinga, P. Roosjen, H. Bartholomeus, and G. Ruessink, PLoS ONE, 9 (2014).

10. D.B. Lobell, and G.P. Asner, Soil Science Society of America Journal, 66 (2002).

11. M. Knadel, F. Deng, A. Alinejadian, L. Wollesen de Jonge, P. Moldrup, and M.H. Greve, Soil Science Society of America Journal, 78 (2014).

12. M. Sadeghi, S.B. Jones, and W.D. Philpot, Remote Sensing of Environment, 164 (2015).

13. J. Tian, and W.D. Philpot, Remote Sensing of Environment, 169 (2015).

14. F. Parera, N.M. Pinyol and E.E. Alonso, Canadian Geotechnical Journal (2019) [Manuscript submitted for publication]

15. D. Hillel, Environmental soil physics (1998). 